\title{
AVALIAÇÃO DOS EVENTOS ADVERSOS A MEDICAMENTOS NO CONTEXTO HOSPITALAR
}

\author{
Evaluation of adverse drug events in the hospital context \\ Evaluación de los eventos adversos a medicinas en el contexto hospitalar
}

Keroulay Estebanez Roque ${ }^{1}$

Enirtes Caetano Prates Melo²

\section{RESUMO}

Este estudo teve como objetivo avaliar a ocorrência de eventos adversos a medicamentos em um hospital público e cardiológico, localizado no município do Rio de Janeiro e classificar os eventos adversos em relação à gravidade do dano. Trata-se de uma investigação baseada em revisão retrospectiva de prontuários. Foi analisada uma amostra aleatória de 112 prontuários de pacientes hospitalizados no período dezembro de 2007 a fevereiro de 2008. Enfermeiras revisoras selecionaram prontuários com potencial evento adverso a medicamento e um grupo de avaliadores confirmou a ocorrência dos eventos adversos e classificaram de acordo com o dano. A incidência de eventos adversos a medicamentos foi de 14,3\%. Em 31,2\% dos casos em que foi detectado o evento houve necessidade de intervenção para o suporte de vida. A detecção de eventos adversos nas instituições hospitalares possibilita conhecer falhas no sistema de medicação, bem como implementar estratégias para reduzi-las.

Palavras-chave: Erros de medicação. Qualidade da assistência à saúde. Gerenciamento de segurança. Enfermagem.

\begin{abstract}
This study aimed to evaluate the occurrence of adverse events in a public cardiology hospital, located in the municipality of Rio de Janeiro and classify adverse events in relation to the severity of the damage. This is an investigation based on retrospective review of medical records. We analyzed a random sample of 112 medical records of patients hospitalized from December 2007 to February 2008. Nurse reviewers selected records with potential adverse drug events and a group of evaluators confirmed the occurrence of adverse events and classified according to the damage. The incidence of adverse drug events was $14.3 \%$. In $31.2 \%$ of cases in which the event was detected, intervention was needed to support life. The detection of adverse events in hospitals enables to know failures medication as well as implement strategies to reduce them.
\end{abstract}

Keywords: Medication errors. Quality of health care. Safety management. Nursing.

\section{Resumen}

Este estudio tuvo como objetivo evaluar la ocurrencia de eventos adversos en un hospital público de medicina y cardiología, ubicado en el municipio de Rio de Janeiro, y clasificar los eventos adversos en relación con la gravedad de los daños. Se trata de una investigación basada en una revisión retrospectiva de las historias clínicas. Se analizó una muestra aleatoria de 112 historias clínicas de pacientes hospitalizados entre diciembre de 2007 y febrero 2008. Las enfermeras revisoras eligieron los registros con potencial de eventos adversos por medicamentos y un grupo de evaluadores confirmaron la ocurrencia de eventos adversos y se clasifican de acuerdo al daño. La incidencia de eventos adversos por medicamentos fue de $14,3 \%$. El $31,2 \%$ de los casos en que se detectó la intervención, era necesario hacerla para mantener la vida. La detección de eventos adversos en los hospitales permite descubrir errores en la administración de los medicamentos, así como aplicar estrategias para reducirlos.

Palavbras-clave: Errores de medicación. Calidad de la atención de la salud. Administración de la seguridad. Enfermería.

\footnotetext{
Enfermeira. Doutoranda em Enfermagem e Biociências pela Universidade Federal Estado do Rio de Janeiro - UNIRIO. Enfermeira do Hospital Universitário Clementino Fraga Filho - UFRJ. Rio de Janeiro - RJ. Brasil. E-mail: keroulay@gmail.com; ${ }^{2}$ Enfermeira. Doutora em Ciências pela Escola Nacional de Saúde Pública - FIOCRUZ. Professora Adjunta do DESP/EEAP/Universidade Federal do Estado do Rio de Janeiro-RJ. Rio de Janeiro - RJ. Brasil. E-mail: enirtes@globo.com
} 


\section{INTRODUÇÃO}

Os eventos adversos a medicamentos (EAMs) ocorridos na assistência medicamentosa podem aumentar o tempo de internação hospitalar, gerar complicações no quadro clínico do paciente e até contribuir para o óbito. ${ }^{1-4}$

EAM é definido pela Organização Mundial de Saúde ${ }^{5}$ como qualquer ocorrência médica desfavorável, que pode ocorrer durante o tratamento com um medicamento, mas que não possui, necessariamente, relação causal com esse tratamento.

A frequência de EAMs tem mostrado uma grande variação na literatura em relação à natureza e complexidade de técnicas utilizadas na identificação e na variabilidade das estimativas dos eventos. A incidência de eventos em alguns estudos internacionais foi muito próxima das ocorridas no Brasil. ${ }^{4-8}$ No Rio de Janeiro, dois importantes estudos identificaram a ocorrência de EAMs, com frequências entre $5,5 \%^{3}$ e $15,6 \%$.

0 risco de ocorrência de um evento adverso não é distribuído igualmente entre os pacientes, e o mesmo indivíduo pode sofrer múltiplos eventos durante o período de internação hospitalar. Os principais fatores de risco associados a esses eventos são idade avançada, comorbidades, polifarmácia, uso inapropriado de medicamentos e ilegibilidade nas prescrições. ${ }^{2,9-11}$ Esta última representa um indicador da qualidade da assistência em saúde e um fator que pode desencadear erros na administração do medicamento e, consequentemente, dano ao paciente.

Os pacientes em uso de medicamentos potencialmente perigosos, como anticoagulantes, opioides, insulina e sedativos, possuem um risco aumentado para evento adverso. ${ }^{11}$ Falhas no sistema de medicação aumentam a probabilidade de eventos adversos relacionados com esses medicamentos. Sendo assim, avaliação das condições que promovem e previnem as falhas e a criação de barreiras de defesa no sistema de medicação podem contribuir na prevenção e redução de danos gerados aos pacientes. ${ }^{11-12}$

A identificação de eventos adversos contribui para o dimensionamento dos erros e falhas ocorridos nos processos de cuidar e é informação valiosa para avaliar a segurança do paciente e da qualidade do cuidado prestado. . $2-13^{-13}$

0 cuidado prestado aos pacientes hospitalizados é complexo e requer que seja executado com qualidade e sem gerar danos desnecessários ao indivíduo. No ambiente hospitalar, a terapia medicamentosa é amplamente utilizada para tratamento das doenças e manutenção da saúde. No entanto, os pacientes hospitalizados e que fazem uso de múltiplos medicamentos encontram-se mais vulneráveis a ocorrência de eventos adversos. Desta forma, tornar-se fundamental o dimensionamento dos EAMs ocorridos durante o período de internação hospitalar. Este estudo tem como objetivos avaliar a ocorrência de EAMs em um hospital cardiológico e classificar os eventos adversos em relação à gravidade do dano.

\section{METODOLOGIA}

Trata-se de um estudo retrospectivo sobre a ocorrência de EAMs realizado em um hospital público, especializado em cardiologia (denominado Hospital Y), localizado no Município do Rio de Janeiro. A instituição de alta complexidade possui em torno de 170 leitos e oferece serviços de investigação diagnóstica e tratamento das doenças cardiovasculares; compõe a rede de hospitais sentinelas, que atua nas áreas de farmacovigilância, tecnovigilância, hemovigilância e vigilância de saneantes.

Realizou-se a identificação e análise dos EAMs através de adaptação do método proposto pelo Institute for Healthcare Improvement $(\mathrm{HHI})^{13}$, baseado em uma revisão retrospectiva de prontuários orientada por critérios explícitos de rastreamento.

0 universo do estudo,composto por pacientes internados no hospital relacionado no período de dezembro de 2007 a fevereiro de 2008. Foram incluídos pacientes maiores de 18 anos que permaneceram internados por pelo menos 48 horas. Foram excluídos pacientes que estivessem em tratamento oncológico.

Foram elegíveis para o estudo 551 prontuários, sendo extraída uma amostra aleatória simples de 112 prontuários, estimada a partir dos seguintes critérios: erro de 10\%, significância estatística de $95 \%$, prevalência do evento estudado de $10 \%$ e perdas de $10 \%$. A seleção aleatória dos prontuários foi realizada a partir da listagem mensal de alta, obtida junto ao Serviço de Admissão e Alta do hospital.

Para esse estudo foi desenvolvido um programa computacional de EAM, destinado ao armazenamento de dados relativos ao rastreamento e à avaliação de eventos. Foram utilizados 21 critérios de rastreamento de EAM, adaptados da lista original. ${ }^{13-14}$ Destes critérios, 12 estão relacionados ao uso de medicamentos; 5 , às alterações nos exames laboratoriais que possam ser associados ao uso de medicamentos; e 4, aos possíveis sinais e sintomas apresentados pelos pacientes após o uso de determinado medicamento registrado pelos profissionais de saúde. Os critérios foram investigados no sumário de alta, nos exames laboratoriais, nas prescrições de medicamentos e nas evoluções da equipe de profissionais.

Para coleta dos dados, adotou-se procedimento de dupla revisão de prontuários por duas enfermeiras revisoras independentes. A presença de pelo menos um critério garantia a seleção do prontuário para um segundo processo de avaliação. Na segunda etapa do processo de avaliação, um médico, um farmacêutico e uma enfermeira avaliaram a ocorrência dos eventos adversos a medicamentos detectados anteriormente, através de uma avaliação implícita. A confirmação do evento adverso e a classificação do dano foram estabelecidas através 
da estratégia de consenso destes especialistas. 0 dano decorrente de um EAM foi classificado de acordo com o Conselho de Coordenação Nacional para Registro e Prevenção de Erros associados ao uso de Medicamentos (National Coordinating Council for Medication Error Reporting and Prevention - NCC MERP). ${ }^{15}$ A NCC MERP ${ }^{15}$ classifica os erros associados ao uso de medicamentos em 9 categorias ( $\mathrm{A}$ a I), sendo que somente as categorias E, F, $\mathrm{G}, \mathrm{H}$ e I estão relacionadas com erros que ocasionaram dano.

Uma vez identificado um possível EAM, sinalizado por um critério de rastreamento, foi realizada nova avaliação do prontuário com a finalidade de confirmar a ocorrência do evento através de evidências clínicas e da relação de causalidade do evento, determinando assim em que momento o evento ocorreu no sistema de medicação. As divergências foram resolvidas por consenso a partir do julgamento clínico.

0 banco de dados foi gerado em programa computacional MS-Access, desenvolvido para essa pesquisa. Para a análise estatística descritiva, os dados inseridos no banco de dados criado foram analisados com o auxílio do pacote estatístico Statistical Package for the Social Sciences (SPSS for Windows, versão 13.0, SPSS Inc., Chicago, IL, EUA).

0 projeto de pesquisa foi submetido e aprovado através do Protocolo nº 0192/22.02.2008 pelo Comitê de Ética e Pesquisa do hospital investigado. Não há conflito de interesses no estudo.

\section{RESULTADOS}

Foram estudados 112 pacientes elegíveis internados no período sob investigação. Verificou-se uma proporção mais alta de mulheres (57,9\%); a média de idade foi 59 anos, com desvio padrão 15,82. Não foi possível analisar as variáveis relacionadas à raça/etnia e ao nível de escolaridade pela alta proporção de informação ignorada. Das internações, metade foi eletiva e, ainda assim, o tempo médio de internação foi elevado, 18,27 dias (desvio-padrão 20,18), variando de 2 a 138 dias.

Do total de pacientes avaliados, 78 (69,6\%) foram identificados como pacientes que apresentaram potenciais EAMs, ou seja, apresentaram pelo menos um critério de rastreamento positivo. Nesses pacientes foram rastreados 238 critérios relacionados à possibilidade de ocorrência de eventos adversos (Figura 1). Dos casos investigados, foram confirmados $16(14,3 \%)$ EAMs. Em um dos casos estudados ocorreram dois eventos adversos (lesão renal e hipoglicemia).

0 evento hipoglicemia, relacionado ao uso de insulina e hipoglicemiantes orais, foi detectado através da aplicação de dois critérios de rastreamento: nível de glicose sérica / capilar e administração de glicose hipertônica. 0 rastreador "glicose $<50 \mathrm{mg} / \mathrm{dL}$ " detectou 5 eventos adversos relacionados à ocorrência de hipoglicemia, e o critério administração de "glicose hipertônica" identificou 4 dos 5 episódios detectados (Tabela 1).

Eventos relacionados à alteração da coagulação sanguínea foram responsáveis por: um caso de hemorragia no pós-operatório de troca valvar e outro após a realização de circulação extracorpórea, um caso de hemorragia em pericárdio, dois casos de hematúria e dois pacientes com hematomas pelo corpo (Tabela 1).

Dos três casos de hemorragia, dois foram identificados através da administração de "sulfato de protamina" e um foi rastreado simultaneamente pela "retirada abrupta de medicação", "transferência para a Unidade de Terapia Intensiva ou Semi-Intensiva" e pelo "INR (Razão Normalizada Internacional) maior que 6" (Tabela 1).

0 evento hematoma foi identificado através dos seguintes rastreadores: "vitamina K", "INR maior que 6", "PTT (Tempo de Tromboplastina Parcial) maior que 100 segundos" e "retirada abrupta de medicação". Em um dos casos identificaram-se hematomas pelo corpo devido à administração de varfarina e, no outro, o evento adverso estava relacionado ao uso de tirofiban, clopidrogel e ácido acetilsalicílico.

0 rastreador "retirada abrupta da medicação" permitiu identificar dois casos de pacientes com hematúria relacionada à administração de antiagregantes plaquetários. Em um caso foram administrados enoxaparina e ácido acetilsalicílico e, no outro, enoxaparina e clopidrogel.

Foram identificados dois casos de arritmia cardíaca a partir dos rastreadores "retirada abrupta de medicação" e "nível de digoxina $>2 \mathrm{mcg} / \mathrm{dL}$ e uso de digoxina". A ocorrência de prurido em membros superiores e dorso, relacionada à administração de contraste radiológico, foi detectada pelo o critério uso de "anti-histamínico". Verificou-se um caso de lesão renal rastreado por "creatinina $>1,5 \mathrm{mg} / \mathrm{dL}$ " e "retirada abrupta da medicação" (Tabela 1).

Em $31,2 \%$ dos casos com EAM foi necessária intervenção para o suporte de vida. Os três eventos hemorragias relacionados ao uso de heparina, enoxaparina e varfarina exigiram uso de aminas vasoativas e reposição hídrica. 0 evento hematoma generalizado devido ao uso de varfarina foi indicado à administração de vitamina $\mathrm{Ke}$ infusão de hemoderivados. No evento fibrilação atrial foi indicada administração de antiarrítmico. Em 50\% dos casos, os eventos adversos ocasionaram um dano temporário aos pacientes e houve a necessidade de tratamento. Os eventos hipoglicemias relacionados a administração de glibenclamida, bradicardia e lesão renal prolongaram a hospitalização e geraram danos temporários ao paciente $(18,8 \%)$ (Tabela 2). 
Figura 1. Estimativa da ocorrência de eventos adversos a medicamentos (EAM) detectados em pacientes internados em um hospital do Rio de Janeiro, dezembro de 2007 a fevereiro de 2008.

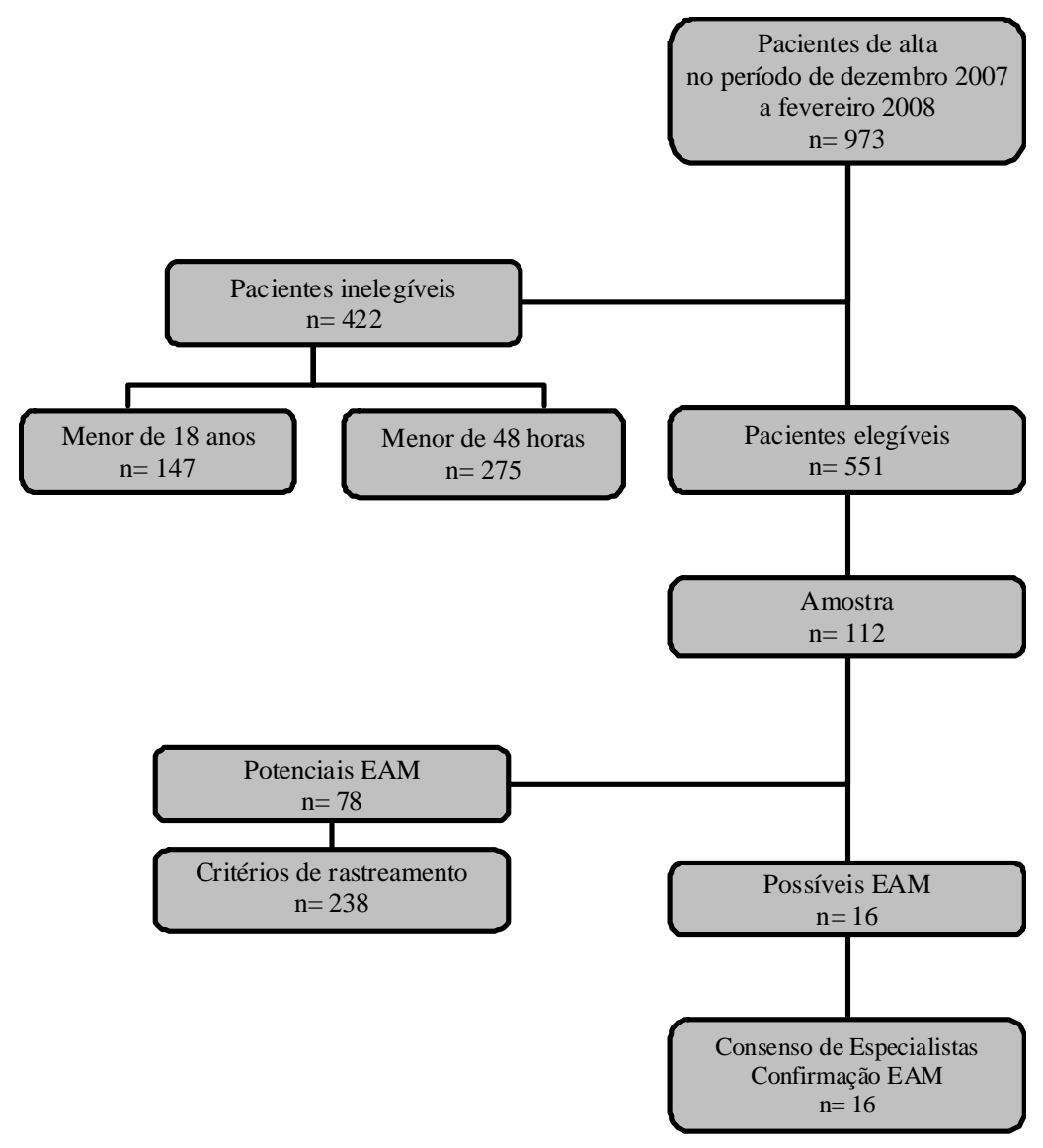

Tabela 1. Distribuição de eventos adversos a medicamentos segundo os critérios de rastreamento, Hospital Y, dezembro de 2007 a fevereiro de 2008.

\begin{tabular}{lcl}
\multicolumn{1}{c}{ Eventos adversos a medicamento } & Frequência & \multicolumn{1}{c}{ Medicamento suspeito } \\
\hline Hipoglicemia & 5 & $\begin{array}{l}\text { Glibenclamida } \\
\text { Metformina } \\
\text { Insulina }\end{array}$ \\
Hemorragia no pós-o peratório de troca de valva mitral & 1 & Heparina \\
Hemorragia após a circulação extracorpórea & 1 & Heparina \\
Hemorragia em pericárdio & 1 & Enoxaparina \\
Hematoma generalizado & 1 & Varfarina \\
Varfarina & Tirofiban \\
Hematoma em região coxofemural direita & 1 & $\begin{array}{l}\text { Ácido acetilsalicílico } \\
\text { Enoxaparina }\end{array}$ \\
Hematúria & & $\begin{array}{l}\text { Clopidrogel } \\
\text { Ácido acetilsalicílico }\end{array}$ \\
Bradicardia & 2 & Digoxina \\
\hline Fibrilação atrial & & Digoxina \\
\hline Prurido em membros superiores e dorso & 1 & Contraste radiológico \\
Lesão renal & 1 & Espirolactona \\
\hline
\end{tabular}


Tabela 2. Número de eventos adversos a medicamentos segundo a classificação da NCC MERP ${ }^{1}$ após a avaliação dos especialistas

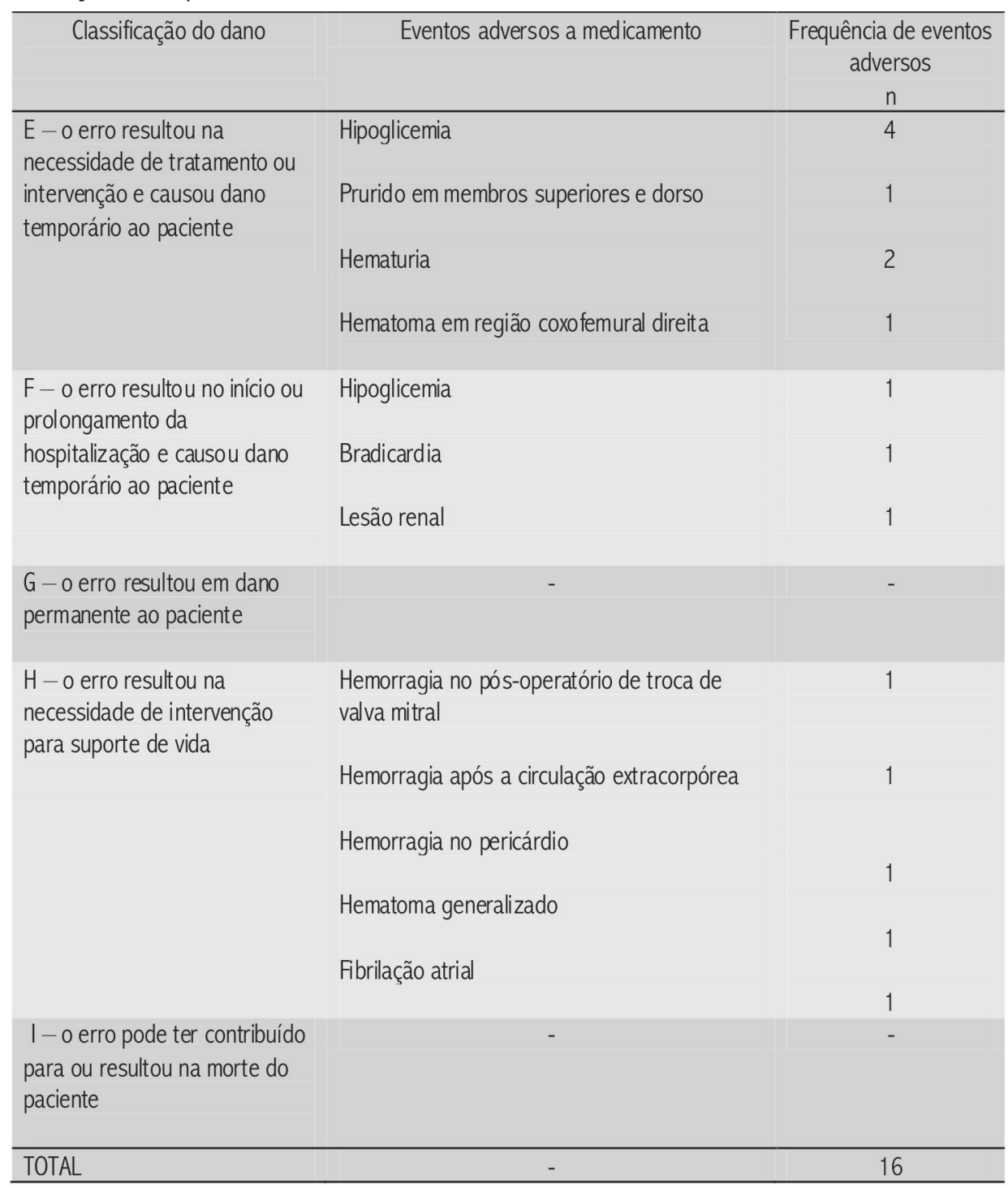

1 National Coordinating Council for Medication Error Reporting and Prevention

\section{DISCUSSÃO}

Observou-se uma incidência de $14,3 \%$ de eventos adversos, e, em $31,2 \%$ dos casos, houve a necessidade de intervenção para suporte de vida. A incidência de EAM descrita nesse estudo é similar à encontrada em outros estudos. ${ }^{1,6,8}$ um estudo piloto realizado em um hospital público e geral, no município do Rio de Janeiro, identificou $15,6 \%$ de EAMs. Um estudo de Rozenfeld et al ${ }^{1}$ foi baseado na identificação dos eventos adversos através de critérios rastreadores muito próximos aos utilizados nesse estudo.

Em 2007, Mendes et $a^{3}$ realizou pesquisa em um hospital geral e de ensino da cidade do Rio de Janeiro e detectou 5,5\% de EAM. No entanto, a pesquisa não teve foco na identificação de EAMs, o que pode explicar a incidência menor de eventos.

A dificuldade de comparabilidade entre os estudos é um aspecto que necessita de maior discussão. Ainda que haja ajuste na gravidade dos casos e o método utilizado para a detecção de eventos seja o mesmo, as especificidades dos processos de cada hospital e o julgamento subjetivo para estabelecer à ocorrência do evento adverso dificultarão a comparação entre os estudos. Sendo assim, a identificação e avaliação dos EAMs nas instituições hospitalares deveriam ser realizadas ao longo do tempo; e as comparações, em diferentes momentos no mesmo hospital.

Além disso, outro fator que dificulta a comparação entre os estudos é a diversidade de terminologias utilizadas para definição do EAM e ao dano. Há necessidade de terminologia padronizada em relação aos termos que envolvem eventos adversos. $^{2}$

Os eventos adversos estive-ram relacionados com a administração de hipoglicemiantes orais, insulina, anticoagulantes, antiagregantes plaquetários, digitálico, contraste radiológico e diurético. 0 Instituto de Segurança Medication Practices (ISMP) ${ }^{12}$ descreve que os anticoagulantes, 
hipoglicemiantes orais e insulina são medicamentos potencialmente perigosos, isto é, possuem um risco inerente de lesar o paciente quando existe falha no processo de administração. Assim, os enfermeiros, responsáveis pela administração desses medicamentos, necessitam monitorar atentamente a ocorrência de eventos adversos.

0 método de identificação de EAMs proposto pelo $\mathrm{IH} \mathrm{I}^{13}$ foi considerado de alta sensibilidade e alta especificidade, sendo recomendado no ambiente hospitalar com objetivo de reduzir a incidência de eventos adversos ao longo do tempo. ${ }^{16} \mathrm{~A}$ detecção de eventos adversos nas instituições hospitalares possibilita 0 conhecimento de falhas no sistema de medicação e a implementação de estratégias destinadas à redução da taxa de eventos, bem como à prevenção da ocorrência do evento e promoção da segurança do paciente e à qualidade do cuidado de enfermagem.

A utilização desse método de rastreamento possui limitações: a qualidade do registro representa um obstáculo no processo de identificação de possíveis eventos adversos; a restrição de dados no prontuário interfere na determinação da causalidade do evento. Note-se que a identificação do evento adverso depende da avaliação implícita realizada pela equipe de especialistas, isto é, a avaliação depende da experiência e do conhecimento do enfermeiro, médico e farmacêutico.

Os enfermeiros e os demais profissionais de saúde tendem a valorizar a descrição de eventos adversos que comprometem a vida do paciente, como os casos de hemorragias e arritmias cardíacas identificados nesse estudo. Todavia, os eventos hipoglicemias não são registrados no prontuário do paciente. Uma glicemia menor do que $50 \mathrm{mg} / \mathrm{dL}$, por exemplo, é um evento que pode ocasionar danos cerebrais irreversíveis ao paciente; mesmo assim o registro da conduta realizada é deficiente, em alguns casos, não existe. Acredita-se que seja baixa a probabilidade de que glicemia menor do que $50 \mathrm{mg} / \mathrm{dL}$ não tenha gerado alguma conduta de enfermagem. Se alguma medida foi tomada, os fatos registrados no prontuário não ficam claros e são incompletos. A enfermagem assume participação fundamental no controle rigoroso dos níveis de glicose sanguínea, bem como na identificação dos sinais e sintomas de hipoglicemia e na administração de glicose hipertônica para reverter esse quadro.

Alterações na coagulação sanguínea relacionadas ao uso de heparina e antiagregantes plaquetários foram identificadas em sete pacientes. As doenças tromboembólicas estão, frequentemente, atreladas às doenças cardíacas, sendo tratadas com drogas que impedem a agregação plaquetária ou que inibem diversas etapas da cascata de coagulação. Os profissionais de saúde, em especial os enfermeiros, devem estar em alerta para episódios de sangramentos como epistaxe, equimose, hematêmese, hematúria e melena. O PTT deve ser monitorizado até que seja determinada a posologia adequada do medicamento anticoagulante. Alterações do PTT podem estar associadas a sangramento por uso de anticoagulante injetável. ${ }^{17}$ Nos casos estudados, o uso de heparina para realização de cirurgias cardíacas aumenta o risco para a ocorrência desse evento.

A intoxicação por digitálico é um tipo de evento adverso amplamente conhecido pelos enfermeiros e demais profissionais de saúde. Mesmo assim, foram detectados dois eventos relacionados a intoxicação por digitálico, no qual um dos eventos necessitou de intervenção com medidas para suporte de vida. A identificação de sinais e sintomas são fundamentais para a prevenção desse tipo de evento, bem como a mensuração dos níveis plasmáticos de digoxina para a determinação da dose diária do medicamento e o monitorização dos valores de

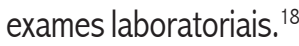

A metade dos EAMs resultou em dano temporário e na necessidade de tratamento (categoria E). Em estudo americano realizado pelo $\mathrm{IHI}$, verificou-se que $79,9 \%$ dos EAMs foram classificados como dano temporário. ${ }^{13}$ Note-se que a proporção de eventos adversos em que houve a necessidade de intervenção para suporte de vida foi maior que o estudo do $\mathrm{IHI}$ $\left(31,2 \%\right.$ e $4,7 \%{ }^{13}$, respectivamente). Esses eventos contribuíram com o aumento dos dias de internação e com o agravamento do quadro clínico, comprometendo a segurança e a vida dos pacientes.

É relevante destacar que o método de revisão retrospectiva de prontuários requer um treinamento bem estruturado e avaliação do desempenho do grupo de revisores. Os revisores devem possuir experiência clínica, conhecimentos em farmacologia e dispensarem um tempo para a investigação do evento. A necessidade de contar com profissionais com tal perfil trouxe durante o trabalho de campo algumas dificuldades. Outras dificuldades enfrentadas foram a falta de ordenação cronológica dos prontuários, difícil legibilidade e uso de abreviaturas nas evoluções dos profissionais de saúde e o tempo dispensado para a aquisição dos prontuários ao serviço de documentação médica.

\section{CONCLUSÕES}

A incidência observada de eventos adversos a medicamento sugere que esta seja ainda maior em outros contextos que não partilhem do nível de excelência da instituição pesquisada. Certamente a incidência de eventos adversos corresponde apenas à 'ponta de um iceberg'. A implantação do método de identificação de EAM a revisão do sistema de medicação na instituição estudada favorecem o monitoramento e a implementação de mecanismos de defesa, barreira e proteção voltadas para a melhoria da segurança do paciente.

0 método proposto pelo IHI favorece a detecção de alguns eventos graves em relação aos de menor gravidade. Os eventos que comprometem a vida do paciente são registrados com maior atenção e detalhamento pelos profissionais de saúde, facilitando assim sua identificação no prontuário. Esse fato pode justificar que aproximadamente um terço dos casos com o evento adverso necessita de intervenção para o suporte de vida. 


\section{REFERÊNCIAS}

1. Rozenfeld S, Chaves SMC, Reis LGC, Martins M, Travassos C, Mendes W, et al . Efeitos adversos a medicamentos em hospital público: estudo piloto. Rev Saude Publica 2009 out; 43(5): 887-90.

2. Cano FG, Rozenfeld S. Adverse drug events in hospitals: a systematic review. Cad Saude Publica. 2009; 25 (supl 3): S360-S372.

3. Mendes W, Martins M, Rozenfeld S, Travassos C. The assessment of adverse events in hospitals in Brazil. Int I Qual Health Care. 2009; 21: 279-84.

4. Senst BL, Achusim LE, Genest RP Cosentino LA, Ford, CC, Little JA, et al. Practical approach to determining costs and frequency of adverse drug events in a health care network. Am J Health Syst Pharm 2001; 58: 1126 132.

5. Mycyk MB, McDaniel MR, Fotis MA, Regalado J. Hospital wide adverse drug events before and after limiting weekly work hours of medical residents to 80. Am J Health Syst Pharm. 2005; 62: 1592- 595.

6. Davies EC, Green CF, Taylor S, Williamson PR, Mottram DR, Pirmohamed $M$. Adverse drug reactions in hospital in-patients: a prospective analysis of 3695 patient-episodes. PLoS One 2009; 4: e4439.

7. Davies EC, Green CF, Mottram DR, Pirmohamed M. Adverse drug reactions in hospital in-patients: a pilot study. J Clin Pharm Ther 2006; 31: 335-341.

8. Dormann H, Neubert A, Criegee-Rieck M, Egger T, Radespiel-Tröger M, Azaz-Livshits T, et al. Readmissions and adverse drug reactions in internal medicine: the economic impact. J Intern Med. 2004; 255: 653-63.

9. Melo ABR, Silva LD. Segurança na terapia medicamentosa: uma revisão bibliográfica. Esc Anna Nery. 2008; 12 (1): 166-72.

10. Zhang M, Holman CDA, Price SD, Sanfilippo FM, Preen DB, Bulsara M. Comorbidity and repeat admission to hospital for adverse drug reactions in older adults: retrospective cohort study. British Medical Journal 2009; 338a: 2752.

11. Gimenes FRE, Teixeira TCA, Silva AEBC, Optiz SP, Mota MLS, Cassiani SHDB. Influência da redação da prescrição médica na administração de medicamentos em horários diferentes do prescrito. Acta Paul. Enferm. 2009; 22(4): 380-84.

12. Federico F. Preventing harm from high-alert medications. Jt Comm J Qual Patient Saf. 2007; 33(9): 537-42.

13. Rozich JD, Haraden CR, Resar RK. Adverse drug event trigger tool: a pratical methodology for measuring medication related harm. Qual Saf Health Care 2003; 12(3): 194-200.

14. Roque KE, Melo ECP. Adaptação dos critérios de avaliação de eventos adversos a medicamentos para uso em um hospital público no Estado do Rio de Janeiro. Rev Bras Epidemiol. 2010; 13(4): 607-19.
15. National Coordinating Council for Medication Error Reporting and Prevention [homepage na Internet]. Rockville: Taxonomy of medication errors. 2009 [cited 2009 Ago 10]. Available from:http://www.nccmerp.org/ aboutMedErrors.html

16. Classen DC, Resar R, Griffin F, Federico F, Frankel T, Kimmel N, Whittington JC, Frankel A, Seger A, James BC. Global trigger tool' shows that adverse events in hospitals may be ten times greater than previously measured. Health Aff, Millwood, 2011; 30(4): 581-89.

17. Majerus PW, Tollefsen DM. Coagulação sanguínea e anticoagulants, trombolíticos e fármacos antiplaquetários. In: Bruton LL, Lazo JS, Parker KL. Goodman \& Gilman: as bases farmacológicas da terapêutica. Rio de Janeiro: McGraw-Hill; 2006. p.1321-339.

18. Rang HP, Dale MM, Ritter JM, Flower RJ. 0 coração. In: Rang HP, Dale MM, Ritter JM, Flower RJ. Rang \& Dale: farmacologia. Rio de Janeiro: Elsevier; 2007. p. 277-97. 